\title{
A NOTE ON MATRICES WITH POSITIVE DEFINITE REAL PART
}

\author{
DAVID LONDON
}

\begin{abstract}
A lemma characterizing complex matrices with positive definite real part is given. It is shown that many results concerning such matrices are straightforward consequences of this lemma.
\end{abstract}

Let $A$ be an $n \times n$ complex matrix, let $H(A)=\frac{1}{2}\left(A+A^{*}\right)$ be its "real part" and let $S(A)=\left(A-A^{*}\right) / 2 i$ be its "imaginary part". Denote by $\Pi_{n}$ the set of all $n \times n$ matrices $A$ for which $H(A)$ is positive definite. A matrix $D$ is strictly dissipative if $D=i A$ for $A \in \Pi_{n}$.

\section{LEMMA. $A \in \Pi_{n}$ if and only if}

$$
A=T \operatorname{diag}\left(1+i \alpha_{1}, \ldots, 1+i \alpha_{n}\right) T^{*},
$$

where $\alpha_{j}, j=1, \ldots, n$, are real numbers and $T$ is a nonsingular matrix.

Proof. If $A$ is given by (*), then $H(A)=T T^{*}$ is positive definite and so $A \in \Pi_{n}$. If $A \in \Pi_{n}$, (*) follows using the theory of pencils of quadratic forms [4, p. 313].

From (*) follows: $D$ is strictly dissipative if and only if

$$
D=T \operatorname{diag}\left(i+\alpha_{1}, \ldots, i+\alpha_{n}\right) T^{*},
$$

where $\alpha_{j}, j=1, \ldots, n$, are real numbers and $T$ is a nonsingular matrix.

The lemma was used long ago by Ostrowski and Taussky [7] and Taussky [8] and more recently by S. Friedland [3]. It was not used nor mentioned however in a series of papers [1], [2], [5], [6] dealing with matrices belonging to $\Pi_{n}$ and with dissipative matrices, although most of the results obtained in these papers are straightforward consequences of the lemma. The purpose of this note is to revive this simple and somewhat forgotten lemma by applying it to derive some of these results, and by this showing its effectiveness in connection with matrices belonging to $\Pi_{n}$ or dissipative matrices.

For $A \in \Pi_{n}$, the representation (*) yields straightforward representations of various matrices connected with $A$. We bring now the representation of some of these matrices.

Let $A \in \Pi_{n}$ be represented by

$$
A=T \operatorname{diag}\left(1+i \alpha_{1}, \ldots, 1+i \alpha_{n}\right) T^{*} .
$$

Then

(a) $A^{*} \in \Pi_{n}$ and

$$
A^{*}=T \operatorname{diag}\left(1-i \alpha_{1}, \ldots, 1-i \alpha_{n}\right) T^{*} .
$$

Received by the editors May 14, 1980.

1980 Mathematics Subject Classification. Primary 15A57. 
(b) $A$ is nonsingular, $A^{-1} \in \Pi_{n}$ and

$$
A^{-1}=\left(D T^{-1}\right)^{*} \operatorname{diag}\left(1-i \alpha_{1}, \ldots, 1-i \alpha_{n}\right) D T^{-1},
$$

where $D=\operatorname{diag}\left(1 /\left(1+\alpha_{1}^{2}\right)^{1 / 2}, \ldots, 1 /\left(1+\alpha_{n}^{2}\right)^{1 / 2}\right)$.

(c) $A^{-1} A^{*}$ is similar to a unitary matrix and

$$
A^{-1} A^{*}=\left(T^{*}\right)^{-1} \operatorname{diag}\left(\frac{1-i \alpha_{1}}{1+i \alpha_{1}}, \ldots, \frac{1-i \alpha_{n}}{1+i \alpha_{n}}\right) T^{*}
$$

(d) $A^{*} A^{-1}$ is similar to a unitary matrix and

$$
\begin{aligned}
A^{*} A^{-1} & =T \operatorname{diag}\left(\frac{1-i \alpha_{1}}{1+i \alpha_{1}}, \ldots, \frac{1-i \alpha_{n}}{1+i \alpha_{n}}\right) T^{-1} . \\
I+A^{-1} A^{*} & =\left(T^{*}\right)^{-1} \operatorname{diag}\left(\frac{2}{1+i \alpha_{1}}, \ldots, \frac{2}{1+i \alpha_{n}}\right) T^{*}
\end{aligned}
$$

(f) $H(A)=T T^{*}$.

(g) $S(A)=T \operatorname{diag}\left(\alpha_{1}, \ldots, \alpha_{n}\right) T^{*}$.

(h) $[H(A)]^{-1} S(A)=\left(T^{*}\right)^{-1} \operatorname{diag}\left(\alpha_{1}, \ldots, \alpha_{n}\right) T^{*}$.

Using the notation of [6], let $m=m(A)=\min _{j} \operatorname{Re}\left(\lambda_{j}\right)$ and let $T=T(A)=$ $\max _{j}\left(\left|\mu_{j}\right|\right)$, where $\lambda_{j}, j=1, \ldots, n$, are the eigenvalues of $A^{-1} A^{*}$ and $\mu_{j}, j=$ $1, \ldots, n$, are the eigenvalues of $[H(A)]^{-1} S(A)$. By (c) and $(\mathrm{h})$,

$$
m=\min _{j}\left(\left(1-\alpha_{j}^{2}\right) /\left(1+\alpha_{j}^{2}\right)\right) \text { and } T=\max _{j}\left|\alpha_{j}\right| \text {. }
$$

Hence,

$$
m=\left(1-T^{2}\right) /\left(1+T^{2}\right),
$$

and we got Theorem 3 of [6]. All the other results of [6] follow easily, using the representations of the various matrices given by (a)-(h). The results of [5], excluding $\S \mathrm{V}$, and the results of [1] and [2] concerning matrices in $\Pi_{n}$ or dissipative matrices follow too. In particular, all the inertia theorems of [1] and [2] for matrices in $\Pi_{n}$ or for dissipative matrices turn out to be results on the inertia of linear combinations of matrices having, by the representations (a)-(h), simultaneous congruent diagonalizations.

\section{REFERENCES}

1. K. Fan, Generalized Cayley transforms and strictly dissipative matrices, Linear Algebra Appl. 5 (1972), 155-172.

2. __ On real matrices with positive definite symmetric component, Linear and Multilinear Algebra 1 (1973), 1-4.

3. S. Friedland, Matrices satisfying the van der Waerden conjecture, Linear Algebra Appl. 8 (1974), 521-528.

4. F. R. Gantmacher, The theory of matrices. I, Chelsea, New York, 1959.

5. C. R. Johnson, An inequality for matrices whose symmetric part is positive definite, Linear Algebra Appl. 6 (1973), 13-18.

6. , Inequalities for a complex matrix whose real part is positive definite, Trans. Amer. Math. Soc. 212 (1975), 149-154. 
7. A. M. Ostrowski and O. Taussky, On the variation of the determinant of a pasitive definite matrix, Indag. Math. 13 (1951), 383-385.

8. O. Taussky, A determinantal inequality of H. P. Robertson, J. Washington Acad. Sci. 47 (1957), 263-264.

Departmant of Mathematics, University of Brttish Columbia, Vancouver, British Columbia V6T 1W5, CaNADA

Department of Mathematics, Technion, Israel Institute of Technology, hafi, Israel (Current address) 